\title{
Defective hMSH2/hMLH1 protein expression is seen infrequently in ulcerative colitis associated colorectal cancers
}

\author{
L Cawkwell, F Sutherland, H Murgatroyd, P Jarvis, S Gray, D Cross, N Shepherd, D Day, \\ P Quirke
}

\begin{abstract}
Background-Ulcerative colitis is associated with an increased risk of colorectal cancer above that of the normal population. The relative risk correlates with the extent and duration of the disease but the genetic basis of ulcerative colitis associated cancer risk is not known.

Aims-To assess the prevalence of microsatellite instability and mismatch repair gene abnormalities in ulcerative colitis associated colorectal cancer.

Patients-Forty six patients with colorectal cancer, with a previous histological diagnosis of ulcerative colitis.

Methods-The frequency of microsatellite instability and/or immunohistochemical expression of hMSH2 and hMLH1 was assessed. Thirty three cases were investigated using both approaches.

Results-Although 6/41 (14.6\%) cases showed microsatellite instability at one or more markers, only one case $(2.4 \%)$ exhibited high level instability (at least two markers affected). Of 38 cases which were assessed using antibodies against hMSH2 and hMLH1, only one case $(2.6 \%)$ showed loss of expression. This case, which showed loss of hMSH2 expression, was the same case which exhibited high level microsatellite instability. The 33 cases which were investigated using both approaches showed that loss of expression of either hMSH2 or hMLH1 was not seen in any case which exhibited microsatellite instability in no more than one marker. Conclusions-This study suggests that both high level microsatellite instability and loss of expression of hMSH2/hMLH1 are infrequent events in ulcerative colitis associated colorectal cancers. Low level microsatellite instability was not associated with loss of expression of either hMSH2 or hMLH1.

(Gut 2000;46:367-369)
\end{abstract}

Keywords: ulcerative colitis; colorectal cancer; microsatellite instability; mismatch repair; inflammatory bowel disease

Department of Histopathology, Torbay Hospital, Torquay, UK D Day

Correspondence to: Dr L Cawkwell

Accepted for publication 18 August 1999

Hereditary non-polyposis colorectal cancer (HNPCC) is an autosomal dominant condition caused by a defect in the DNA mismatch repair pathway, with the majority of cases having germline mutations in the hMSH2 or hMLH1 genes. ${ }^{1}$ Such mutations often lead to the production of a truncated protein. ${ }^{1}$ In somatic tissue the second functional copy of the gene may then be inactivated either by allele loss, hypermethylation of the promoter, or further mutation. Errors at DNA replication are corrected via the DNA mismatch repair pathway and any defect within this system will lead to the rapid accrual of genome wide mutations. Tumours which have defective mismatch repair show a characteristic molecular phenotype, termed microsatellite instability (MI), ${ }^{2}$ as repeated DNA sequences (microsatellites) are often replicated incorrectly. If the mismatch repair system does not correct these errors then these regions of DNA can become extended or shortened in relation to the original template. HNPCC tumours characteristically show a high level of altered microsatellite sequences (over $29 \%$ of microsatellites tested would be affected ${ }^{2}$ ) and are also termed replication error (RER) positive. Approximately $10 \%$ of sporadic colorectal cancers are also RER positive ${ }^{34}$ and patients with these carcinomas appear to have a better prognosis than patients whose carcinoma is RER negative. ${ }^{3-5}$

The inflammatory bowel disease ulcerative colitis is associated with an increased risk of colorectal cancer above that of the normal population. ${ }^{6}$ The relative risk has been shown to correlate with the extent and duration of disease $^{78}$ but the genetic basis of ulcerative colitis associated cancer predisposition is poorly understood. MI has been found to be a feature of some ulcerative colitis associated colorectal cancers ${ }^{9-11}$; however, the reported incidence varies from $21 \%{ }^{10}$ to $40 \% .{ }^{11}$ High level MI (two or more markers affected), which is a feature of HNPCC tumours, has been reported in $8-40 \%$ of ulcerative colitis associated colorectal cancers. ${ }^{10}{ }^{11}$ It is not clear whether the increased cancer risk in some patients with ulcerative colitis may be owing to a genetic predisposition such as a variant type of HNPCC.

It has been reported that low, and even high level MI may be seen in non-neoplastic mucosa of patients with ulcerative colitis without a colorectal carcinoma ${ }^{11}$ and in chronic inflammatory conditions such as pancreatitis. ${ }^{12}$ The finding of $\mathrm{MI}$ in $85 \%$ of patients with ulcerative colitis with high grade dysplasia and in $60 \%$ with no dysplasia lead Brentnall and
Abbreviations used in this paper: HNPCC, hereditary non-polyposis colorectal cancer; MI, microsatellite instability; RER, replication error. 
colleagues $^{11}$ to suggest that this may represent the saturation of the DNA repair process owing to continuous cell regeneration associated with chronic inflammation. Furthermore, it was suggested ${ }^{11}$ that this could possibly account for the increased risk of colorectal cancer that is associated with ulcerative colitis, owing to an increased frequency of uncorrected errors. It has been reported that polymorphisms of both hMSH2 and hMLH1 are associated with ulcerative colitis or ulcerative colitis related cancer risk. ${ }^{13}{ }^{14}$ It has been suggested that ulcerative colitis may stress a suboptimal mismatch repair system in patients with a particular hMSH2 phenotype, thus giving rise to uncorrected mutations and increasing the risk of cancer. ${ }^{13}$ In this scenario uncorrected mutations could occur within DNA mismatch repair genes leading to loss or reduction of this repair function. A further theory suggests that folate deficiency, which is seen in some patients with long standing ulcerative colitis, could contribute to aberrant DNA replication and repair. ${ }^{15}$

We and others have previously shown that sporadic colorectal carcinomas which exhibit high level MI (RER positive) have lost expression of either the hMSH2 or hMLH1 proteins. ${ }^{16}{ }^{17}$ For hMLH1 in particular this may be owing to hypermethylation of the promoter region. ${ }^{18}{ }^{19}$ In ulcerative colitis associated colorectal cancers the status of these proteins has not been studied.

We investigated a series of 46 ulcerative colitis associated colorectal cancers using a combination of fluorescence MI assays and immunohistochemical analysis of $\mathrm{hMSH} 2$ and hMLH1. We aimed to confirm the frequency of low and high level MI in ulcerative colitis associated colorectal cancers and to determine whether these cancers show a higher prevalence of MI than sporadic cases. This may support a theory of inflammation causing continuous tissue regeneration and saturation of mismatch repair leading to uncorrected errors, possibly enhanced by a deleterious genetic polymorphism. High level MI may indicate a possible causative link with a variant HNPCC phenotype. We also aimed to assess the status of the hMSH2 and hMLH1 proteins in these cancers. The incidence of loss of expression of these proteins may again indicate whether ulcerative colitis cancer predisposition could be owing to variant HNPCC. Finally, we aimed to correlate the status of hMSH2/hMLH1 with the MI results.

\section{Materials and methods}

PATIENTS

Forty six colorectal adenocarcinomas were assessed from 46 patients with a previous histopathological diagnosis of ulcerative colitis.

PCR BASED MICROSATELLITE INSTABILITY ASSAY Following histological assessment and microdissection, DNA was extracted from formalin fixed, paraffin wax embedded normal (uninvolved large/small bowel or cancer free lymph node) and carcinoma tissue for each patient using a rapid method developed for use with the Nucleon DNA extraction kit (Scotlab Ltd,
Strathclyde, UK). ${ }^{20}$ A fluorescence multiplex MI assay, as described previously, ${ }^{21}$ was used with a panel of four markers: D5S346, ${ }^{22}$ p53(2), ${ }^{23}$ DCC (2), ${ }^{23}$ and RB1. ${ }^{23}$ If at least two markers showed MI the carcinoma was classified as RER positive.

IMMUNOHISTOCHEMICAL STAINING OF hMSH2 AND hMLH1

One block of formalin fixed, paraffin wax embedded adenocarcinoma tissue was selected per case. Wherever possible, this block included a region of non-neoplastic mucosa adjacent to the carcinoma. Immunohistochemical analysis was performed using monoclonal antibodies against hMSH2 (NA27, Calbiochem, Nottingham, UK) and hMLH1 (13291A, Pharmingen, San Diego, California, USA) as described previously. ${ }^{16}$ All results were scored without knowledge of the MI results. Loss of expression was recorded when nuclear staining was seen in non-neoplastic tissue but was completely absent in adjacent malignant cells. ${ }^{16}$

\section{Results}

Forty six ulcerative colitis associated colorectal carcinomas were assessed from 46 patients. Five cases were suitable only for immunohistochemical analysis owing to the lack of sufficient tissue for DNA extraction. Eight cases were only analysed using the MI assay as the blocks were unavailable when the immunohistochemical assays were performed. Thirty three cases were investigated using both approaches. Although 6/41 (14.6\%) cases showed MI at one or more marker, only one case $(2.4 \%)$ exhibited high level instability (at least two markers affected). From a total of 38 cases which were assessed using antibodies against hMSH 2 and hMLH1 only one case $(2.6 \%)$ showed loss of expression. This case, which showed loss of expression of hMSH2, was the same case which exhibited high level MI. The 33 cases which were investigated using both approaches showed that loss of expression of either hMSH2 or hMLH1 was not seen in any case which was MI negative (28 cases) or exhibited MI in only one marker (four cases).

\section{Discussion}

Our findings suggest that, although MI is a feature of a subset of ulcerative colitis associated colorectal cancers, high level MI (at least two markers affected) is seen infrequently. Our results are similar to those of Suzuki and colleagues ${ }^{10}$ who reported frequencies of $21 \%$ and $8 \%$ for low and high level MI respectively in a series of 68 ulcerative colitis associated cancers. The report by Brentnall and colleagues $^{11}$ which describes a finding of high frequency $\mathrm{MI}$ in $40 \%$ of ulcerative colitis associated colorectal cancers may have been an overestimate owing to the use of only five cases and the scoring of MI based on an autoradiographic approach.

The frequency of MI which we have detected in ulcerative colitis associated colorectal cancers is not dissimilar to that seen in sporadic colorectal cancers, ${ }^{3416}$ and may be slightly 
lower. This suggests that saturation of the mismatch repair system may not be a causative factor in the majority of ulcerative colitis associated cancers. The low incidence of high level MI also suggests that a variant HNPCC phenotype is unlikely to be responsible for the increased risk of colorectal cancer in most patients with ulcerative colitis.

Immunohistochemical analysis of hMSH2 and hMLH1 expression in 38 cases revealed a low frequency of defects in these major mismatch repair proteins. This again suggests that a variant type of HNPCC which is based on germline mutations in $\mathrm{hMSH} 2 / \mathrm{hMLH} 1$ is not a major cause of ulcerative colitis associated cancers.

Loss of expression of hMSH2 was associated with the single case which showed high level MI. This 34 year old patient had no family history of colorectal cancer and presented with a Dukes's stage C1 adenocarcinoma of the proximal colon 24 years after the onset of ulcerative colitis.

We have previously reported the association between high level MI and loss of hMSH2/ hMLH1 expression in sporadic colorectal cancers. ${ }^{16}$ Low level MI (one marker only affected) was not associated with loss of expression of either hMSH2 or hMLH1 in sporadic colorectal cancers ${ }^{16}$ and the same finding was noted for four ulcerative colitis associated cancers in this study. This suggests that low level MI may be associated with incomplete loss of expression of $\mathrm{hMSH} 2 /$ hMLH1, or loss of expression of a different mismatch repair enzyme-for example, hPMS1, hPMS2, hMSH6/GTBP, or hMSH3. Alternatively, low level MI could be the result of saturation of the mismatch repair system. ${ }^{11}$

In conclusion, this study suggests that both high level MI and loss of expression of $\mathrm{hMSH} 2 / \mathrm{hMLH} 1$ are infrequent events in ulcerative colitis associated colorectal cancers. The incidence of $\mathrm{MI}$ is similar to that seen in sporadic colorectal cancers but may be lower in ulcerative colitis associated colorectal cancers.

This work was supported by Yorkshire Cancer Research.

1 Liu B, Parsons R, Papadopoulos N, et al. Analysis of mismatch repair genes in hereditary non-polyposis colorectal cancer patients. Nat Med 1996;2:169-74.
2 Aaltonen LA, Peltomaki P, Leach FS, et al. Clues to the pathogenesis of familial colorectal cancer. Science 1993; 260:812-16.

3 Lothe RA, Peltomaki P, Meling GI, et al. Genomic instability in colorectal cancer: relationship to clinicopathological variables and family history. Cancer Res 1993;53:5849-52.

4 Bubb VJ, Curtis LJ, Cunningham C, et al. Microsatellite instability and the role of $\mathrm{hMSH} 2$ in sporadic colorectal cancer. Oncogene 1996;12:2641-9.

5 Thibodeau SN, Bren G, Schaid D. Microsatellite instability in cancer of the proximal colon. Science 1993;260:816-19.

6 Mellemkjaer L, Olsen JH, Frisch M, et al. Cancer in patients with ulcerative colitis. Int f Cancer 1995;60:330-3.

7 Ekbom A, Helmick C, Zack M, et al. Ulcerative colitis and colorectal cancer. A population-based study. $N$ Engl F Med 1990;323:1228-33.

8 Gillen CD, Walmsley RS, Prior P, et al. Ulcerative colitis and Crohn's disease: a comparison of the colorectal cancer risk in extensive colitis. Gut 1994;35:1590-2.

9 Kern SE, Redston M, Seymour AB, et al. Molecular genetic profiles of colitis-associated neoplasms. Gastroenterology 1994;107:420-8.

10 Suzuki H, Harpaz N, Tarmin L, et al. Microsatellite instability in ulcerative colitis-associated colorectal dysplasias and cancers. Cancer Res 1994;54:4841-4.

11 Brentnall TA, Crispin DA, Bronner MP, et al. Microsatellite instability in nonneoplastic mucosa from patients with chronic ulcerative colitis. Cancer Res 1996;56:1237-40.

12 Brentnall TA, Chen R, Lee JG, et al. Microsatellite instability and K-ras mutations associated with pancreatic adenocarcinoma and pancreatitis. Cancer Res 1995;55:4264-7.

13 Brentnall TA, Rubin CE, Crispin DA, et al. A germline substitution in the human MSH2 gene is associated with highgrade dysplasia and cancer in ulcerative colitis. Gastroenterology 1995;109:151-5.

14 Pokorny RM, Hofmeister A, Galandiuk S, et al. Crohn's disease and ulcerative colitis are associated with the DNA repair gene MLH1. Ann Surg 1997;225:718-25.

15 Cravo ML, Albuquerque CM, Salazar de Sousa L, et al. Microsatellite instability in non-neoplastic mucosa of patients with ulcerative colitis: effect of folate supplementation. Am 7 Gastroenterol 1998;93:2060-4.

16 Cawkwell L, Gray S, Murgatroyd $\mathrm{H}$, et al. Choice of management strategy for colorectal cancer based on a diagnostic immunohistochemical test for defective mismatch repair. Gut 1999;45:409-15.

17 Thibodeau SN, French AJ, Cunningham JM, et al. Microsatellite instability in colorectal cancer: different mutator phenotypes and the principal involvement of hMLH1. Cancer Res 1998;58:1713-18.

18 Kane MF, Loda M, Gaida GM, et al. Methylation of the hMLH1 promoter correlates with lack of expression of hMLH1 in sporadic colon tumors and mismatch repairdefective human tumor cell lines. Cancer Res 1997:57:808 11 .

19 Herman JG, Umar A, Polyak K, et al. Incidence and functional consequences of hMLH1 promoter hypermethlation in colorectal carcinome. Proc Natl Acad Sci USA 1998;95:6870-5.

20 Cawkwell L, Quirke P. Comparison and development of DNA extraction methods for formalin-fixed, paraffinembedded tissue [abstract]. F Pathol 1997;182(suppl):22A.

21 Cawkwell L, Li D, Lewis FA, et al. Microsatellite instability in colorectal cancer: improved assessment using fluorescent polymerase chain reaction. Gastroenterology 1995;109: 465-71.

22 Spirio L, Nelson L, Ward K, et al. A CA-repeat polymorphism close to the adenomatous poyposis coli (APC) gene offers improved diagnostic testing for familial APC. Am f Hum Genet 1993;52:286-96.

23 Cawkwell L, Lewis FA, Quirke P. Frequency of allele loss of DCC, p53, RB1, WT1, NF1, NM23 and APC/MCC in colorectal cancer assayed by fluorescent multiplex polymerase chain reaction. Br f Cancer 1994;70:813-18. 